\title{
Die Sammlung
}

\section{,ЯMus Ratur und Geifteswelt"}

nunmebr über 800 Bãnde umfallend, bletet wirllide "Einfübrungen" In abgefdloffene Wiffensgebiete für ben Unterridt oder Selbitunter= ridt des Ealen nad den beutigen metbodifden Anforderungen und ers füllen fo ein Bedütinis, dem weder umfangreide Enzïllopädien, nods flizzenbafte $\mathcal{A b r i f l e ~ e n t f p r e d e n ~ t o ̈ n n e n . ~ D i e ~ B a ̈ n d e ~ w o l l e n ~ j e d e m ~ g e i f t i g ~}$ Mündigen die Möglidleit fめaffen, fid obne befondere Dorkenniniffe an fiderfter Quelle, wie fie die Darftellung ourd betufene Bettreter der $\mathfrak{W}$ iffen=

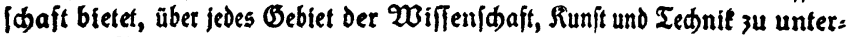
ridten. Sie wollen ibn dabei zugleid uninittelbar im Beruf fördern, Den Beffdistreis etwefternd, die Einfidt in die Bedingungen der Berufsarbeit dertiefend.

Die Sammlung bietet aber aud Dem fadmann eine rafde 3 uver:

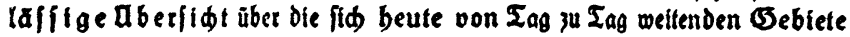
bes geiftigen Eebens in weiteftem Umfang und vermag fo vor allem aud bem immer fĭtrter werdenden Bedürfnis des forfकers 34 dienen, fí

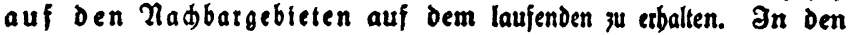
Dienft diefer Aufgaben baben fiф Darum aud in dantenswerter Wetfe von Rnfang an die beften Namen geftellt, getn die Gelegenbeit benukend, Ild an weitefte Rireife zu wenden.

So tonnte der Sammlung aud Der Erfolg nidt feblen. Mebr als Die Galfte ber Bände liegen beteits in 2. bis 8. Ruflage vor, insgejamt bat die Sammlung bis jegt eine Berbreitung oon faft 5 Millionen Exemplaren gefunden.

Hlles in allem find die fdmuden, gebaltvollen Bände bejonders geeignet, die Sreude am Bude 3 meten und batan w gewöbnen, einen Betrag, Den man für Erfüllung törperlider Bedürfnifle niфt anzuleben pflegt, aud für die Beftiedigung geiftiger anzuwenden.

Jeder det meift reids flluftrietten Bände ift in fiф abgefblofien und einzeln tâfliф

Peipzig, im Ruguft 1925.

B. $\mathfrak{G}$. Teubner 


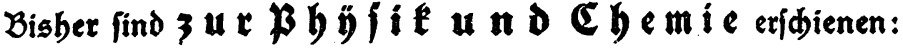

\section{Bhyfif: Cinfübrung, Grundlagen and Sejdidte.}

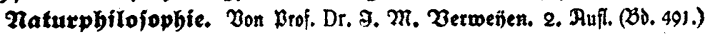

Dte Srunobegriffe dex modernan Tafurlebre. Einfubung in die Bbifit. Von Boirat Brof. Dr. f. A u erbad. 5. Auifl. (230. 40.)


Reg.= Rat $\mathfrak{B}$ tof. Dr. K. $\mathcal{B}$ örnftein. Wit 90 Mbbiloungen. (BD. 371.)

Cinfübrung in Die Relativitätotheoric. Bon Dr. W. Blod. 3., vetb. Ruflage. mit 18 Siguten. (30.618.)

Raturwffenfकaften, Matbematif und Meoizin im elafifiden Altertum. Bon Brof. DP. Job. E. Geiberg. 2. Hufl. Mit 2 Éguren. (BD. 370.)

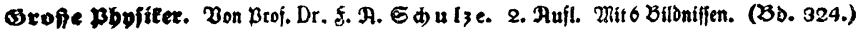

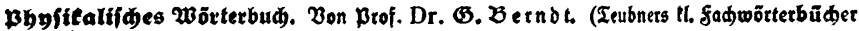
3b. 5.)

\section{Medjanif.}

Meфanit. Bon Brof. Dr. G. Gamel. 3 Bände. (BO. 684/86.) I. Gundbegriffe Der

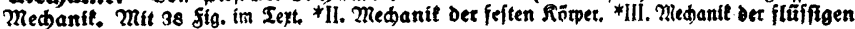
und luftförmigen fổrpet.

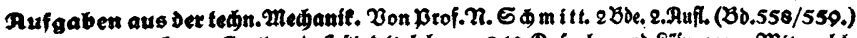

I. BewegungsIebre, Etatif und Sejtigleitslebre. 240 Pufgaben und Eojungen. Mit $3 a b l=$ reiben Sfig. im Tezt.

II. Dñnamit und Bj̈braulit. 2. Hufl. bearb. von Obetfudientat prof. Dr. B. W 198 Pufgaben uno Eöfungen. Mit $3 a b I r$. Figuten im Teft,

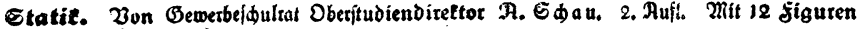
im Iext. (30. 828.)

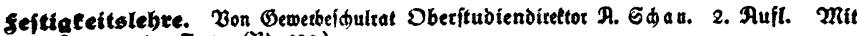
119 Siguren im Teft. (BD. 829.)

\section{Optif, angewandte Optif und Sfrablungsexjळeinunget.}

Das Etळt und die farben. (Ginfübrung in die Optil.) Won Drof.Dr. E. Oraeg. 5. Ruflage. Mif 100 Rbbildungen. (20. 37.)

Eidtbare und unfítbare Eteablen. Bon Geb. KegierungssRat Brof. Dr. $\boldsymbol{R}$.



Oie optifiden Inftumente. (Eupe, Mitroflop, Sernrobr, photograpbildes Objettiv uno

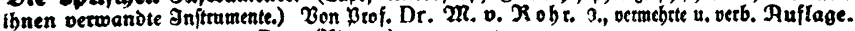
2hit 89 MbbilDungen in Text. (30.38.)

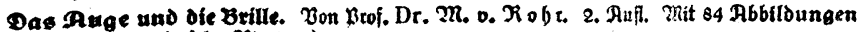
und, Eidhtbrudtafel. (BD. 372.)

Das 2uitrofeop, feine wiffenidaftliden Grundagen und feine Anwendung. Won Dr. A. Ebringbaus. Mit 75 Mbbildungen im Tezt. (BD. 678.)

Cinfübrung in ofe mifrotednif. Bon Brof. Dr. $\mathfrak{3}$. franz und Oberfudiendiretor Dr. G. Б中 nefoer. Mit 18 Rbb. (BD. 765.)

Epettrofeople. Bon Brof. Dr. E. Grebe. 2. 2hufl. Wit 63 figuren im Teat und anf 2 Doppeltafeln. (BD. 284.)

Dfe fifnematograpbie, thte Btundlagen und ibre Anwendungen. Bon Dr. G. Eeb. mann. 2. Huflage von Dr. W. Terté. 2nit 68 zum Ieil neucn Abbild. (30. 358.)

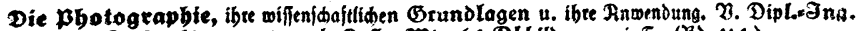
Dit. Dr. O. B relinger. 2., verb. Auf. Mit 64 Mbbildungen. i. T. (30.414.)

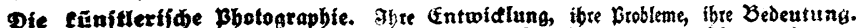
Bon Studientat Dr. W. Mia ritat. 2., vetb. Ruft. Mit Bildetanbang. (BD. 410.)

Die Rönteenftrablen und ibee Ruwendung. Won Dr. med. O. Bu\&̃̃. Wit 94 Abbildungen in Text und auf 4 Iafeln. 2. Derb. Hufl. (30. 556.)

ARuG 13: pbopft und Ebemie. VIII. 25. 


\section{Baxmelebre.}


Dr. $\mathfrak{x}$ Bönftein. 2, Durdggefebene Huflage. Grsg. von Brof. Dr. $\mathcal{H}$. Wigand. Mit 33 Abbilbungen im Tezt. (BD. 172.)

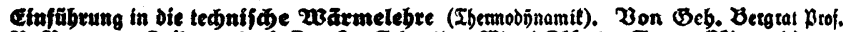

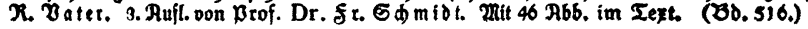

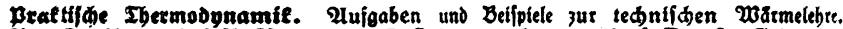

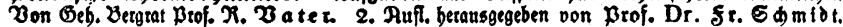
Thit 40 Mbb. im Tezt und 3 Tafeln. (30. 596.)

\section{Cinfubrung in die Cbemie.}

Cinfübrung in ofe allgemefne Cbemie. Bon Stubientat Dr. 8.3 a vint. 2. Huft. 2Mit 24 figuren. (Bo. 582.)

Cinfübrung in Die anorganifde Ebemic. Bon Gtudienrat Dr. B. Bavint. Mit 3) Hbbildungen im Teft. (3D. 598.)

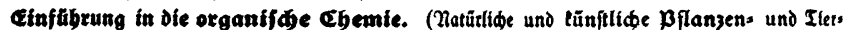
ftoffe.) Won Studientat Dr. $3 . \mathcal{B}$ a vint. 3. Hufl. Mit 9 Abb. im Sezt. (Bb. 187.)

Cinfübrung in de analytfíse Qbemie. Bon Dr. f. Küs berg. 2 Bde. I. Theorie und

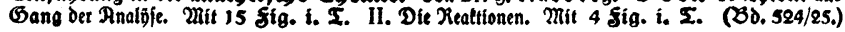
Cinfübrung in die Biodemie in elementaxer Daritellung. Bon Brof. Dr. 20,256 .

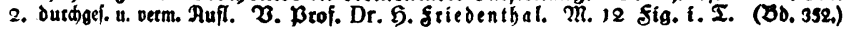
Clettrodemie und ibre Rnwendungen. Bon Brof. Dr. א. A rndt. 2. Auflage. mit 37 Hbbilloungen im SeFt. (BD. 234.)

Das Radium und die Rabioaftipität. Bon Brof. Dr. M. Centne rs 3 wet. 2. Rufl. Mit 33 figuren'im Sext. (BD. 405.)

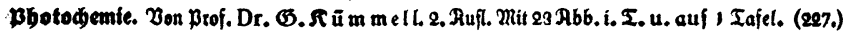
Euft, Wafiex, etøt und wärunc. Ginjübrung in die Experimentaldemie. Bon Geb.

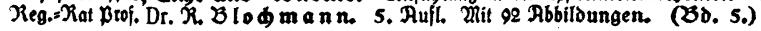

Das Wajfex. Bon (5eb. Regietungstat Dr. D. Anjelinino. Mit 44 Abbftb. (Bb.291.)

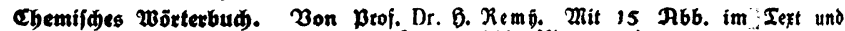

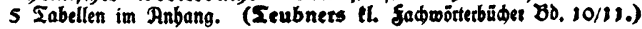

\section{Cbemijळe Tednologie.}

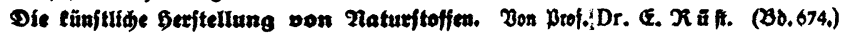
Det Euftitidtoff und feine 2 etwertung. Bon Brof. Dr. R. Faifer, 2. Rufl. mit 13 Hbbiloungen (BD. 3)3.)

Rgrifulturdemie. Pon Dr. B. F rifめe. 2. Detb. Auf. Mit 2 ) Abbildungen. (30. 314.) Die Eprengitoffe, thre Cbemie und Seduologie. Bon Geb. Reg.= Rat Brof. Dr. R. B iedermann. 2. Ruflage. 2Rit 12 Siguten. (30.286.)

Sarben u. Sarbitoffe. Jbre Erzengung u. Betwendung. Bon Dr. A. 3 a t. Mit s) Abb. (30. 483.)

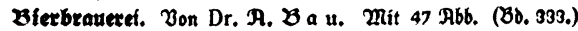

wörteebuक dex Warentunde. Bon Brof. Dr. M. Bietfd. (Teubnets tleine fad. möttetbüđet. BD. 3.)

Raturlebre im gaufe.

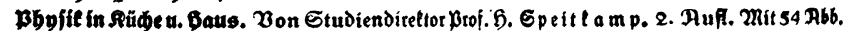
(30.478.)

Cbemie in Rüde und Gaus. Bon Dr. J. Flein. 5. Aufl. (Bo. 76.)

Desinfettion, Efexilfiation, Ronfexviexung. Von Regietungs: und Medizinalrat Dr. O. Eolbtig. mit 20 Rbbiloungen. (30. 401.)

Cenabrung uno Rabeungemittel. Bon Geb. Rat Beof. Dr. $\mathfrak{R}$. 3 a n h. 3. Huf. Whit 6 Hbbildungen und \& Safeln. (Bo. 19.)

Die Bafterien im Gausbalt ber Ratur und des Meniden. Bon Brof. Dr. E. Ontseit. 2. Aufl. Mit 13 मbbiloungen. (BD. 242.)

Weitere Bände befinden fid in Borbereitung. 


\section{Aus $\mathfrak{R a t u r}$ und Geijteswelt}

Sammlung wiffenidaffllids=gemeinverftändlider Darifellungen 76. Band

\section{Cbemíe \\ in $\mathfrak{R} \mathfrak{u}(\mathfrak{c}) \mathfrak{u n d} \mathfrak{G} \mathfrak{a} \mathfrak{s}$ \\ Bon \\ Dr. Ioleph $\mathfrak{R l e i n}$}

fünfte $\mathfrak{A u f l a g e}$

23. bis 27. Taujend

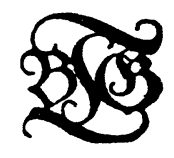

Springer Fachmedien Wiesbaden GmbH 1925 
ISBN 978-3-663-15625-3 ISBN 978-3-663-16200-1 (eBook)

DOI 10.1007/978-3-663-16200-1

Softcover reprint of the hardcover 5th edition 1987

Alle Redte, einfdließlid Des aberfegungstedts, vorbebalten 


\section{Dorwort зur fünften Auflage.}

Das Wejentliḑe, was dieje Auflage won der vorḩergehenden unter= ineidet, ift die Einfügung eines Gbidznittes über die Ditamine in der Themie der Ernährung. Ferner war es nötig, andere Gbichnitte etwas umzugejtalten, damit fie den heutigen Derhältnifjen beljer Rechmung tragen. So 3. B. Find die Seifenerjabjtoffe, die der Krieg gezeitigt hatte und nun überlebt find, ganz unberürfiç̨tigt geblieben, während einige Angaben über die Indanth̨renfarben am Plake waren.

Ih h̆offe, dáz die Änderurgen dem werfhen die freundiche Gufnahme, die es bisher gefunden ḩat, erḩalten werdert.

Mannheim, Guguit 1925.

Dr. Joleph Klein. 


\section{Inhal tsverzeiđnis.}

I. Allgemeine Chemie. Sette

1. Einleitung . . . . . . . . . . . . . . 7

Gggregat3ujtände. Sdhmelzpunft. Eritarrungspunft. Siedepunft. Dejtillation. Spejifildes Gewid̨t. Diffulion. Osmotifher Druf.

2. Die atmoiphärifhe $\mathfrak{L} u f t . \ldots \ldots 11$

Euftoruc. Barometer. Saueritoff. $\mathfrak{D}_{3}$ on. Stifftoff.

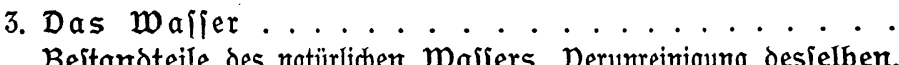
Bejtandteile des natürliđłen Wafjers. Derumreinigung desfelben.
härte. Mineralwäfjer. Eis. Diđhtemarimum. Wajferitoff. Knallgas. Gemifhe. Themilhe Derbindungen. Elemente.

4. Die Elemente................

Dorfommen in der hatur. Eegierungen. Sejte Eöjungen. $\mathfrak{R a}=$ dium. Allotropie. Künttlicher Diamant uno Graphit. Die Sym= bole der Elemente. Gewinnung derjelben. Rub́. Ę̧lor. Sđ̆wefel. Koh̆lenitoff. Zinf. Queffiliber. Kupfer. Meefing. Bronze. Kupfer= münzen. Silber. Silbermünzen. Gold. Golomünzen. Zinn. Blei.

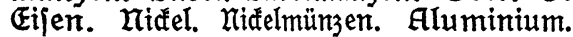

5. Die demifhen Derbindungen . . . . . . . .

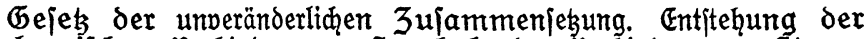
đhemilhyen Derbindungen. Symbole der Derbindungen. Atome und Atomgewidhte. Molefüle und Tolefulargewiđte. Benennung der Derbindungen. Säuren. Bajen. Sal 3 e. Meutrale Derbindungen. Krijtallwaffer. Derwitterung. F్ grometerbilder. Kaljinierte Sal3e.

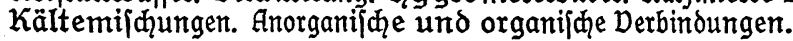

a) Anorganilįe Derbindungen .............

Kiejeliäure. Waljerglas. GIas. Kohlenjäure. Braulepulver.

$\mathrm{Sal}_{3}$ [äure. Sąwefeljäure. Gmmoniaf. Salmiafgeift. Salmiaf. hiriḩhornjal3. Borax. Kodyjal3. Soda. Doppeltfohlenjaures Vatrium. Kaliumoryd. Kalfwaller. Mörtel. 3 ement. Sdwefel= faures Kal 3 ium. Gips. Kal 3 iumfarbid. Korund. Saphir. Rubir. Cöpferwaren. porzellart.

b) Organijđ̨e Derbindungen . . . . . . . . . . . 39

Ätḩy IaIfoḩol. Spiritus. Efligläure. Eflig. Sette und öle. wadsarten. Seifen. Pflajter. Glyzerin. 3uder. Milduzuder. Stärfe. Bügeln. Kollodium. Zelluloid. Gálalith. Bałelite. 
1. $\mathfrak{A b b a} \mathfrak{u}$ und $\mathfrak{A} \mathfrak{u} \mathfrak{b a u}$ der Stoffe ......... 45 Der Stoffwedhjel. Die Atmung. Das Blut. Kahrungsbedürfinis. Das Zujtandefommen der diemijąen Dorgänge.

2. Die Kahrungsmittel. Allgemeines. . . . . . .

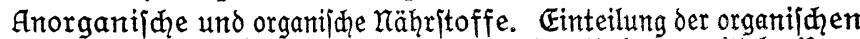


dauung. Gufnahme des Eijens. Serratin.

3. Die Eimeífitoffe.

3ujammenjełung und Einteilung derjelben. Eier. Mith. Käle.

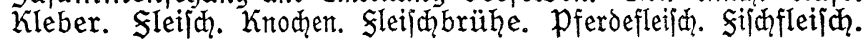

4. Die Sette ................ 59 Butter. Buttermild. Buttererjazmittel.

5. Die Kohlenhyorate ..............

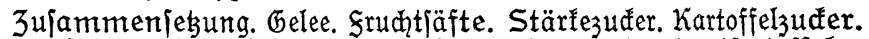
ḩonig. Mehl. Kindermeḩle. Reifen. Süpwerden der Kartoffeln.

6. Die Genubmittel ............... Allgemeines. Spirituoien. Alfoholfreie Getränte. Kaffee. Tee. Katao. Shotolade. Koffeinfreier Kaffee. Kaffeejurrogate. Tabał.

7. Ditamine

Einteilung derjelben. Avitaminojen.

\section{Die Ghemte in ber Küׁle.}

1. Der Kellelitein.

Die Ausid̨eidungen des Walfers. Enthäärtung des Walfers. Weid̨es wajfer.

2. Die 3 ubereitung der Speifen

Kodhen. Düniten. Braten. Güllenfrüđte. Bacten. Altbatenes Brot. Die die Derdaung beeinflufjenden plydiologijhen momente. $3 \mathfrak{u}=$ taten. Sleifhentraft. ḩefenertrafte. Б̆ewürze. Suppenwürze.

3. Die Sermentprozelie............. pepfin. Bittere Mandeln. Meerrettid. Senf. Diajtaje. Invertaje. 3ymaje. Wein. Bier. Maltaje. Brot. Bafpulver. Kumýs. Kefir.

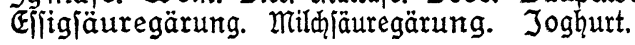

4. Die Konjervierungsmethoden . . ........

Gustrođ̆nen. Kältefonjervierung. Räuđ̆ern. Salzen. pöfeln. Kon= jervierung mit Efjig und 3 ufer. Tarinieren. Konjervierung mit Spiritus. Salizylfäure. Benzoeläure. Sterilifieren. Sorhhletiḑer $\mathfrak{A p}=$ parat. wed = und Bade=Duplerc=Apparat. Konjervierung mit ÖI und Sett. Suppentonjerven. 
5. Die Speifenvergiftungen.

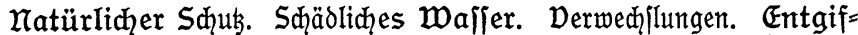
tung giftiger Kahrungsmittel. Kartoffelvergiftung. Dilzpergiftung. ptomaine. Pathogene Bafterien. Sleifhvergiftungen. Danille= eisvergiftungen. Konjerven. Kupfergehalt der Konjerven.

\section{Die Chemie in der woh̆nung.}

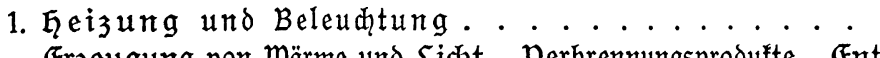
Erzeugung von Wärme und Sid̨t. Derbrenmungsprodufte. Ent= zündungstemperatur. Entflammungspunft. Explofionen. দุeizwert und Eeudhtfraft. Glühlidht. Seuermachen und Seuerlö|chent. Brenn= material. Steinfohle. Detroleum. Dajelin. Stearinferzent. Paraffin. Eeuchtgas. Gzetylengas. Seuergefähnrliche Stoffe.

2. Die Desinfeftionsmethoden . . ....... 106 Die natürliḑe Desinfeftion. Die Fünjtlid̨e Desinfeftion. Desinfef= tionsmittel. Räuḩerungen.

\section{Die Chemie in ber Kleidung.}

1. waidgen und Bleidien.

Dorgang beim waiken. Wert der Seife. Zuläbe beim wainen. Katurbleidhe. Kunjtbleid̨e. Die \{aueritoffabgebenden wajd̨mittel. Bleidhjoda. Bläuen.

2. Das \$ärben der Spinnfajern

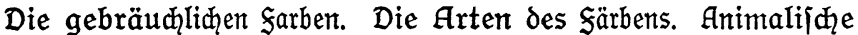
und vegetabilifh̨e Sajern. Särbetheorie. Beurteilung der Sarben. Edhtheit der Sarben. Indantḩrenfarben. দqausfärberet.

3. Die Beleitigung der flefen.

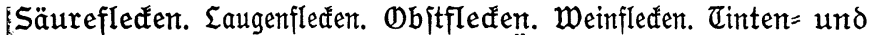

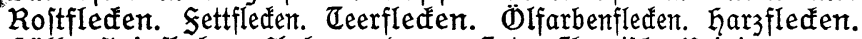

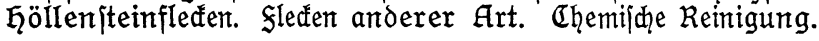

Regijter.................... . 119 